Research Paper

\title{
Berberine Inhibits the Metastatic Ability of Prostate Cancer Cells by Suppressing Epithelial-to-Mesenchymal Transition (EMT)-Associated Genes with Predictive and Prognostic Relevance
}

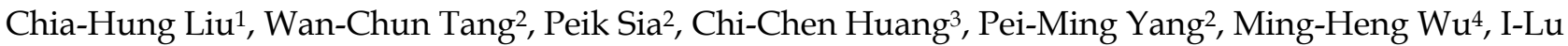 \\ Lai $^{5}$, Kuen-Haur Lee ${ }^{\circledR}$ \\ 1. Department of Urology, Taipei Medical University-Shuang Ho Hospital, New Taipei City, Taiwan; \\ 2. Graduate Institute of Cancer Biology and Drug Discovery, College of Medical Science and Technology, Taipei Medical University, Taipei, Taiwan; \\ 3. The PhD Program for Neural Regenerative Medicine, College of Medical Science and Technology, Taipei Medical University, Taipei, Taiwan; \\ 4. The PhD Program for Translational Medicine, College of Medical Science and Technology, Taipei Medical University, Taipei, Taiwan. \\ 5. Division of Medicinal Chemistry, College of Pharmacy and Comprehensive Cancer Center, The Ohio State University, Columbus, OH, USA.
}

$\square$ Corresponding author: Dr. Kuen-Haur Lee, Graduate Institute of Cancer Biology and Drug Discovery, College of Medical Science and Technology, Taipei Medical University, No. 250 Wu-Hsing Street, Taipei 11031, Taiwan. Tel: +886-2-27361661 ext. 7627, Fax: +886-2-66387537, E-mail: khlee@tmu.edu.tw.

() Ivyspring International Publisher. This is an open-access article distributed under the terms of the Creative Commons License (http://creativecommons.org/ licenses/by-nc-nd/3.0/). Reproduction is permitted for personal, noncommercial use, provided that the article is in whole, unmodified, and properly cited.

Received: 2014.06.25; Accepted: 2014.11.03; Published: 2015.01.01

\begin{abstract}
Background: Over $70 \%$ of cancer metastasis from prostate cancer develops bone metastases that are not sensitive to hormonal therapy, radiation therapy, or chemotherapy. The epithelial-to-mesenchymal transition (EMT) genetic program is implicated as a significant contributor to prostate cancer progression. As such, targeting the EMT represents an important therapeutic strategy for preventing or treating prostate cancer metastasis. Berberine is a natural alkaloid with significant antitumor activities against many types of cancer cells. In this study, we investigated the molecular mechanism by which berberine represses the metastatic potential of prostate cancer.

Methods: The effects of berberine on cell migration and invasion were determined by transwell migration assay and Matrigel invasion assay. Expressions of EMT-related genes were determined by an EMT PCR Array and a quantitative RT-PCR. The prognostic relevance of berberine's modulation of EMT-related genes in prostate cancer was evaluated using Kaplan-Meier survival analysis.

Results: Berberine exerted inhibitory effects on the migratory and invasive abilities of highly metastatic prostate cancer cells. These inhibitory effects of berberine resulted in significant repression of a panel of mesenchymal genes that regulate the developmental EMT. Among EMT-related genes downregulated by berberine, high BMP7, NODAL and Snail gene expressions of metastatic prostate cancer tissues were associated with shorter survival of prostate cancer patients and provide potential therapeutic interventions.

Conclusions: We concluded that berberine should be developed as a pharmacological agent for use in combination with other anticancer drug for treating metastatic prostate cancer.
\end{abstract}

Key words: Berberine; EMT; Prostate cancer.

\section{Introduction}

Advanced prostate cancer is refractory to conventional treatments by anti-hormonal therapy, radiotherapy, and chemotherapy [1]. The first-line sys- temic docetaxel-based chemotherapies used as care for patients with metastatic prostate cancer are only palliative and typically culminate in the death of patients after about 12 19 months [2]. These clinical limitations illustrate the pressing need to utilize new 
and improved molecular indicators of prostate cancer progression. The epithelial-to-mesenchymal transition (EMT) genetic program was implicated as a significant contributor to prostate cancer progression [3]. Recent studies demonstrated that over $70 \%$ of cancer metastasis from prostate cancer develops bone metastases that are not sensitive to hormonal therapy, radiation therapy, or chemotherapy [4]. As such, targeting the EMT represents an important therapeutic strategy for preventing or treating prostate cancer metastasis. Therefore, identifying and developing new molecular therapies to target the EMT of metastatic prostate cancer are urgently needed.

Berberine, a clinically important natural isoquinoline alkaloid, is characterized by a diversity of pharmacological effects [5]. It also shows the suppressive effect of berberine on the proliferation of various cancer cells through induction of cell cycle arrest and cellular apoptosis [6-8]. Recently, the use of berberine has attracted great attention as an alternative anti-metastasis therapy of various lines of cancers such as gastric, oral, bladder, and liver cancers, considering its low toxicity and low cost [9-13]. Very recently, berberine was reported to inhibit the metastatic potential of lung cancer cells through suppression of the transforming growth factor (TGF)- $\beta$-induced EMT [14]. However, little is known about the action of berberine on the migration and invasion of prostate cancer cells which needs to be explored.

In this study, we demonstrated that the migration and invasion of metastatic prostate cancer cells could be inhibited by berberine. We also found the inhibition of migration and invasion of prostate cancer cells through berberibe-regulated suppression of EMT-related genes. In addition, simultaneous high expressions of three berberine-inhibited EMT-related genes, such as bone morphogenetic protein 7 (BMP7), NODAL and Snail, were identified to be survival biomarkers for prostate cancer outcomes.

\section{Materials and Methods}

\section{Chemicals and antibodies}

Berberine $(>98 \%$ purity), DMSO, and 3-(4,5-dimethylthiazol-2-yl)-2,5- diphenyltetrazolium bromide (MTT) were obtained from Sigma-Aldrich (St. Louis, MO, USA). Antibodies against E-cadherin, Vimentin, Twist and Snail were purchased from Cell Signaling Technology (Beverly, MA, USA). The antibody against $\beta$-actin was purchased from Chemicon International (Temecula, CA, USA).

\section{Cell cultures}

PC-3 and LNCaP cell lines (ATCC, Manassas, VA, USA) were cultured in RPMI-1640, supplemented with $10 \%$ fetal bovine serum (FBS) and antibiotics. The DU145 (ATCC) cell line was cultured in minimum essential Eagle's medium supplemented with $10 \%$ FBS, 2 mM L-glutamine, and antibiotics. Cultures were maintained in a $5 \% \mathrm{CO}_{2}$ humidified atmosphere at $37^{\circ} \mathrm{C}$.

\section{Western blot analysis}

Cell lines and human prostate cancer specimens were placed in lysis buffer at $4{ }^{\circ} \mathrm{C}$ for $1 \mathrm{~h}$. Protein samples were electrophoresed using $12 \%$ sodium dodecylsulfate (SDS)-polyacrylamide gel electrophoresis (PAGE), performed as previously described (15).

\section{Cell viability assay}

Cell viability was determined by MTT assays as reported previously (16). Cells were seeded at $10^{4}$ cells/well $24 \mathrm{~h}$ prior to treatment.

\section{Wound-healing assay}

After PC-3 cells had grown to confluence in 6-mm culture plates, an artificial "wound" was created using a 200- $\mu$ l pipette tip to scratch the confluent cell monolayer. The wound area was subsequently inspected after 12 and $24 \mathrm{~h}$ using an inverted phase-contrast microscope with a digital camera. The wound-healing speed was calculated as the percentage of the initial wound until total wound closure at different time points using NIH ImageJ software (US National Institutes of Health).

\section{In vitro migration and invasion assays}

Assays were performed using Falcon ${ }^{\mathrm{TM}}$ cell culture inserts $(8-\mu \mathrm{m}$ pore size) in a 24 -well format (BD Biosciences, San Jose, CA, USA) according to the vendor's instructions. In the migration assay, PC-3 cells $\left(10^{4}\right.$ cells/well) in $0.5 \mathrm{ml}$ of serum-free medium containing berberine at the indicated concentration were seeded onto membranes of the upper chambers, which had been inserted into wells of 24-well plates containing 10\% FBS-supplemented medium. After 12 h, cells were fixed with $100 \%$ methanol and stained with 5\% Giemsa stain (Merck, Darmstadt, Germany). Un-migrated cells that remained in the upper chambers were removed by wiping the top of the insert membranes with a damp cotton swab, which left only those cells that had migrated to the underside of the membranes. The membranes were mounted on glass slides, and numbers of cells in three randomly chosen high-power fields were counted. For the invasion assay, PC- 3 cells $\left(10^{5}\right.$ cells/well) in $0.5 \mathrm{ml}$ of serum-free medium containing berberine at the indicated concentration were seeded onto Matrigel-coated membranes of the upper chambers and incubated at $37^{\circ} \mathrm{C}$. The lower chambers contained the same amount of berberine in 10\% FBS-medium. After $24 \mathrm{~h}$, noninva- 
sive cells remaining on the upper surface of the membranes were removed with a cotton swab. Cells on the lower surface of the membrane were fixed in $100 \%$ methanol and stained with 5\% Giemsa stain for $10 \mathrm{~min}$. Membranes were mounted on glass slides, and numbers of cells in three randomly chosen high-power fields were counted. All experiments were performed three times and photographed under a phase-contrast microscope $(200 \times)$.

\section{EMT polymerase chain reaction (PCR) array and quantitative reverse-transcription (RT)-PCR}

Total RNA was extracted from untreated (control) and berberine-treated PC-3 cells using a Qiagen RNeasy kit and Qiashredder columns according to the manufacturer's instructions (Qiagen, Valencia, CA, USA). One microgram of total RNA was reverse-transcribed to complementary DNA (cDNA) using ReactionReady ${ }^{\mathrm{TM}}$ First Strand cDNA Synthesis Kit (SABiosciences, Frederick, MD, USA) and applied to the EMT PCR Array following SABiosciences' RT-PCR manual (cat. no. PAHS-090Z, 96-well format). Plates were processed in an Applied Biosystems StepOnePlus ${ }^{\mathrm{TM}}$ Real-Time PCR System (Applied Biosystems, Foster City, CA, USA) using an automated baseline and threshold cycle detection. Data were interpreted using SABiosciences' web-based PCR array analysis tool. The quantitative RT-PCR for confirmation of regulated genes was performed as previously described [17]. Sequences of specific primers for each gene are listed in Table 1.

\section{Statistical analysis}

Statistical analyses were performed as recommended by an independent statistician. These included unpaired Student's $t$-test (cell viability, migration and invasion assays, and quantitative RT-PCR). All statistical analyses were performed using SPSS software (SPSS, Chicago, IL, USA), all values are expressed as the mean \pm standard error of the mean (SEM), and statistical significance was accepted at $p<0.05$.

\section{Results}

Berberine suppresses the motility of PC-3 cells

To determine whether berberine can inhibit human prostate cancer metastasis, we first examined the expression levels of EMT mediators in various prostate cancer cell lines with an immunoblot analysis. As shown in Figure 1A, among three prostate cancer cell lines, PC-3 cells were demonstrated to have the highest migratory ability among the three prostate cancer cell lines (18) and with a high expression level of the mesenchymal marker, vimentin, and a low expression level of the epithelial marker, E-cadherin. LNCap was the most static cell line with a low expression level of vimentin and a high expression level of E-cadherin. An increase in cell motility has an impact on the metastatic potential of cancer cells. We thus tested whether berberine could decrease the motility of PC-3 cells, a cell line with high metastatic potential. Before examining cell motility, we performed a cell viability analysis to determine the lowest concentration of berberine that did not induce cell death. Berberine reduced cell viability, effectively inhibiting proliferation at concentrations of $>50 \mu \mathrm{M}$ of three prostate cancer cell lines (Figure 1B). We found that concentrations of berberine of $10 \sim 50 \mu \mathrm{M}$ inhibited cell proliferation by $<20 \%$ after $24 \mathrm{~h}$ of treatment (Figure 1B). We next performed a wound-healing assay in PC-3 cells, in which a line was formed by scratching the cell monolayer with a pipette tip. In this model, the gap was mainly covered by cells that moved to close it rather than cells that proliferated, at least at early time points when cells have not had enough time to proliferate. Results are presented as a percent of the distance that the wound had closed at that particular time point (Figure 1C, 1D). At $12 \mathrm{~h}$, about $40 \%$ of the initial gap had closed in vehicle-treated cells, while $5 \% \sim 20 \%$ of the gap had closed in berberine-treated cells. At $24 \mathrm{~h}$, about $90 \%$ of gap had closed in vehicle-treated cells, while only $5 \% \sim 50 \%$ of the gap had closed in berberine-treated cells (Figure 1C, 1D), suggesting that berberine inhibited their motility. Taken together, these results suggest that berberine inhibited cell motility that resulted in reduced closure of the gap.

Table 1. Quantitative RT-PCR Primer Sets.

\begin{tabular}{lll}
\hline Primer & Sense & Antisense \\
\hline PDGFRB & 5'-TGGGCTAGACACGGGAGAATACT-3' & 5'-GGTGGGATCTGGCACAAAGA-3' \\
COL1A2 & 5'-CACAAGGAGTCTGCATGTCTAAGTG-3' & 5'-CTCTTGTAAAGATTGGCATGTTGCT-3' \\
BMP7 & 5'-CAACCTCGTGGAACATGACAA-3' & 5'-CGTGACAGCTTCCCCTTCTG-3' \\
NODAL & 5'-CATCCGACCAACCATGCATAC-3' & 5'-TCAGCGGCTTGGTCTTCACT-3' \\
WNT11 & 5'-GAAGGACTCGGAACTCGTCTATCT-3' & 5'-CTTGTTGCACTGCCTGTCTTG-3' \\
\hline
\end{tabular}


A

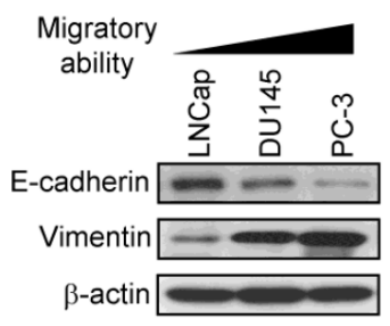

C

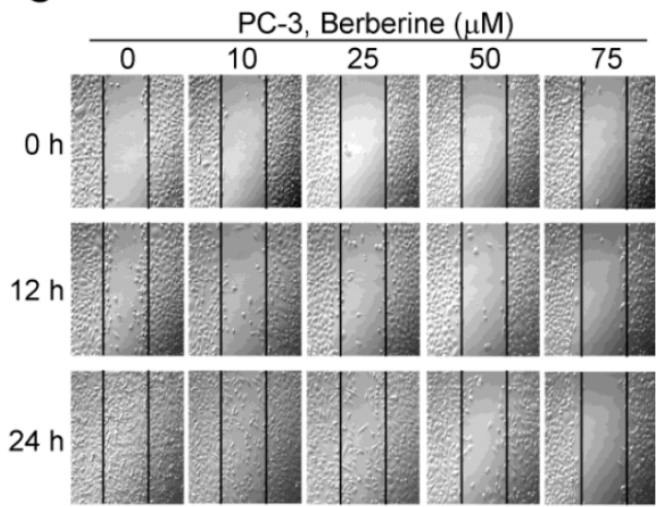

B

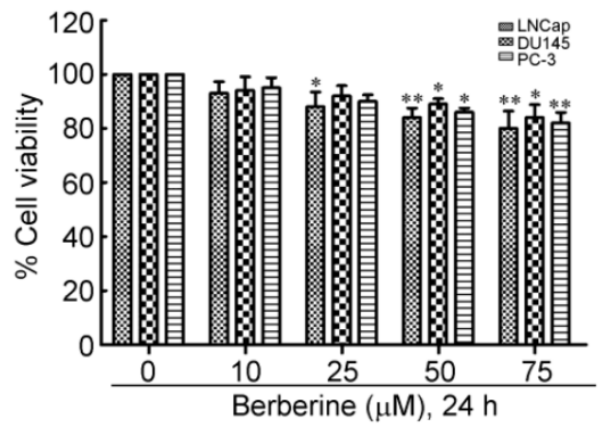

D

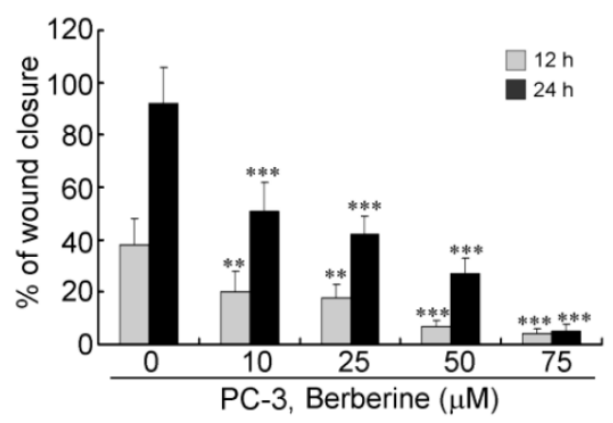

Figure 1. Effect of berberine on prostate cancer cell motility. (A) Western blot analysis of expression levels of the epithelial-to-mesenchymal transition (EMT) inhibitor, E-cadherin, and EMT inducer, vimentin, in a panel of prostate cancer cell lines (LNCaP, DU-145, and PC-3) with different degrees of aggressiveness. (B) Concentration-dependent effects of berberine on cell viability were determined in a panel of prostate cancer cell lines using an MTT cell viability assay. (C) Confluent monolayers of PC-3 cells were scratched using a plastic pipette tip and then incubated in medium in the presence of $0,10,25,50$, and $75 \mu \mathrm{M}$ berberine. At 0 , 12 , and $24 \mathrm{~h}$ after wounding, cells were photographed under a phase-contrast microscope $(100 \times)$ equipped with a CCD camera. (D) The level of cell migration into the wound scratch was quantified as the percentage of wound healing. Columns, average of three independent measurements; bars, SEM. ${ }^{* *} p<0.01,{ }^{* * * *} p<0.001$.

\section{Berberine inhibits migration and invasion of highly metastatic prostate cancer cell lines in a concentration-dependent manner}

Cancer metastasis, a complex and multistep process, is highly related to cell motility, and the migratory and invasive abilities of cancer cells (19). To further determine the influence of berberine in the migration and invasion of prostate cancer cells, PC-3 and DU145 cells were treated with berberine at the indicated concentration. Then the migration transwell assay was performed to determine the migratory abilities of the two prostate cancer cell lines after $24 \mathrm{~h}$ treatment. As shown in Figure 2A, the migratory abilities of these two prostate cancer cell lines were significantly reduced after berberine treatment. The inhibition rate was respectively $30 \%$ and $60 \%$ in PC-3 and DU145 cells compared to the control group at low concentration $(25 \mu \mathrm{M})$ of berberine treatment (Figure $2 \mathrm{~A}$, right panel). Subsequently, the effects of berberine on the invasion of these cells were also determined by matrigel invasion assay system. The invasion capability of berberine-treated groups was inhibited $43 \%$ and $55 \%$ in PC-3 and DU145 cell line respectively, compared to the control group at low concentration $(25$ $\mu \mathrm{M})$ of berberine treatment (Figure 2B). Although exposure of either cell line to berberine within the same dose range caused a dose-dependent reduction in cell viability within $24 \mathrm{~h}$ of treatment (Figure 1B), this drug-mediated inhibition of cell motility and invasion was not attributable to cell death as the rates of the concentration-dependent decreases in invasion and migration were significantly greater than that in viability (Figure 2C for PC-3 cells, $p<0.01$; Figure 2D for DU145 cells, $p<0.005)$. These data show that berberine exerts inhibitory effects on the migratory and invasive abilities of prostate cancer cells.

\section{Inhibition of mesenchymal marker gene expressions in response to berberine}

The role of berberine in regulating EMT was further demonstrated by detection the protein expression of several key EMT effectors, including E-cadherin, vimentin, Twist and Snail. It is noteworthy that berberine had no appreciable effect on the aforementioned biomarkers expression in PC-3 cells (Additional file 1: supplementary Figure 1A), except for Snail. To identify genes associated with the invasive phenotype of prostate cancer cells which are regulated by berberine, we profiled changes to EMT 
genes using an $\mathrm{RT}^{2}$ Profiler ${ }^{\mathrm{TM}}$ Human EMT PCR Array analysis consisting of 84 epithelial and mesenchymal genes (Additional file 1: supplementary Table 1). A 4-fold or greater difference in mRNA expression levels was used as the cut-off to determine significant regulatory effects on genes involved in tumor metastasis. Berberine treatment $(25 \mu \mathrm{M}, 24 \mathrm{~h})$ was found to exclusively downregulate five of 84 EMT-related genes assessed in this experiment (Figure 3A). Remarkably, berberine treatment negatively affected the expression of three mesenchymal genes regulating the developmental EMT: platelet-derived growth factor receptor-beta (PDGFRB), collagen, type I, alpha 2 (COL1A2), and bone morphogenetic protein 7 (BMP7) (Figure 3B). Of note, berberine treatment simultaneously attenuated expressions of the TGF- $\beta$ responsive genes, NODAL and WNT11 (Figure 3B),

A
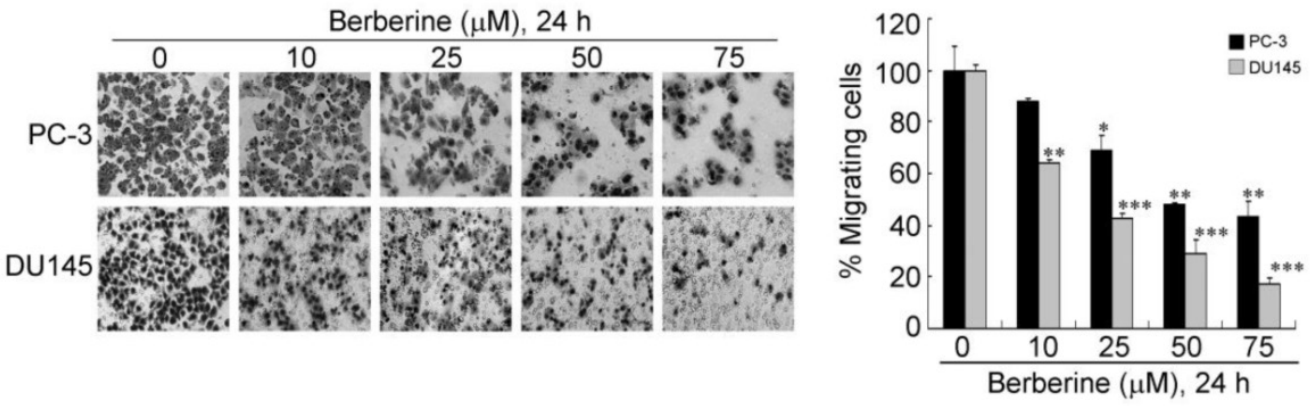

B
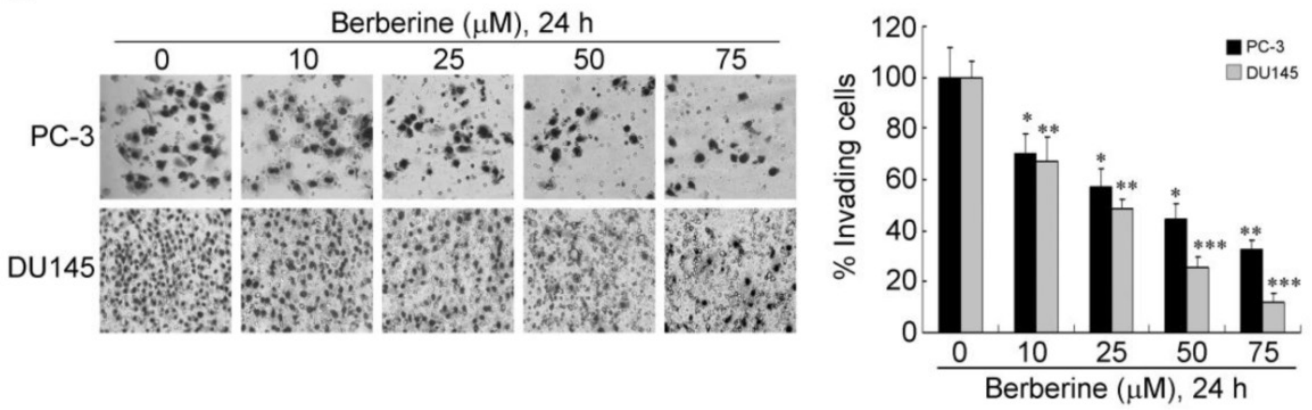

C

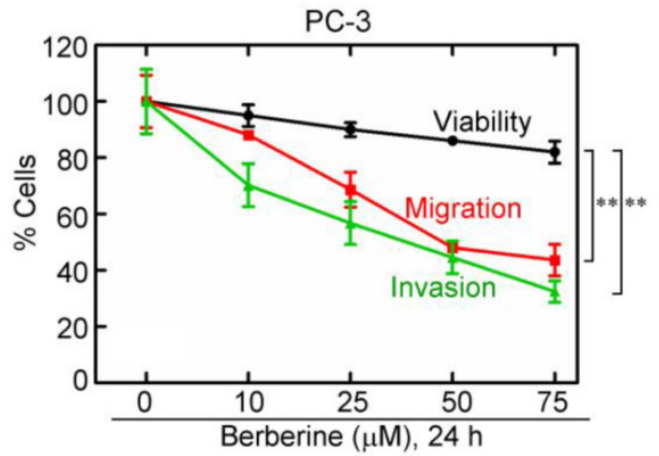

D

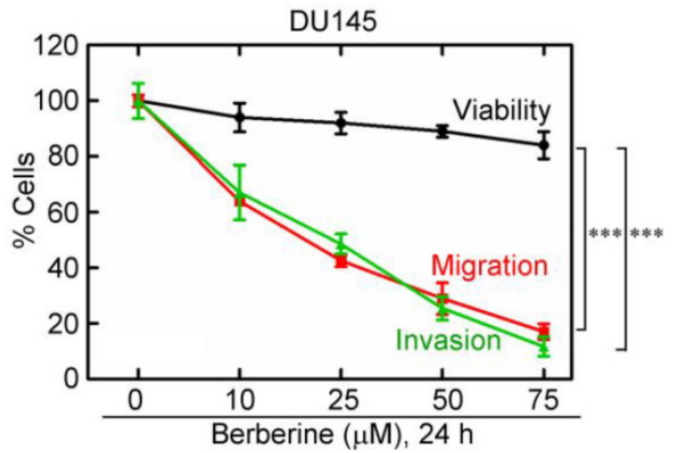

Figure 2. Dose-dependent inhibitory effects of berberine on the migratory and invasive abilities of highly metastatic prostate cancer cells. (A) PC-3 and DU145 cells were treated with berberine $(0,10,25,50$, or $75 \mu \mathrm{M})$ for $24 \mathrm{~h}$ and then subjected to a cell migration analysis as described in "Materials and Methods". (B) PC-3 and DU145 cells were treated with berberine $(0,10,25,50$, or $75 \mu \mathrm{M})$ for $24 \mathrm{~h}$ and then subjected to cell invasion analysis as described in "Materials and Methods" (magnification $\times 200)$. Trend analysis of the rates of concentration-dependent decreases in the viability, migration, and invasion of PC-3 (C) and DU145 (D) cells at the indicated concentrations of berberine. Data represent the mean \pm SEM of three independent experiments. ${ }^{*} p<0.05, * * 0.01,{ }^{* * * *} p<0.001$. 
A

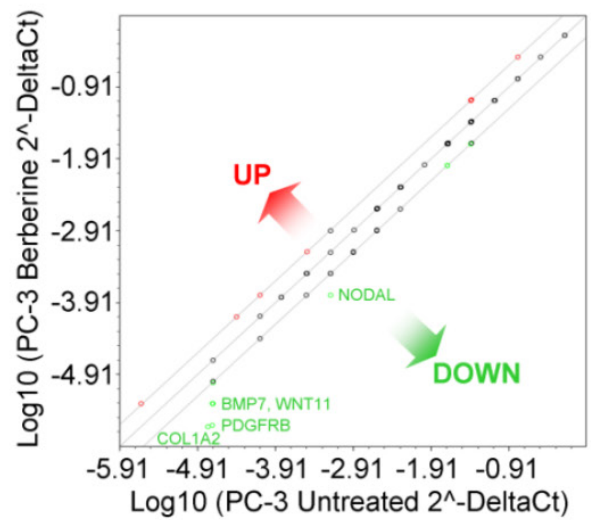

B

$$
\text { Top five downregulated }
$$
EMT-related genes

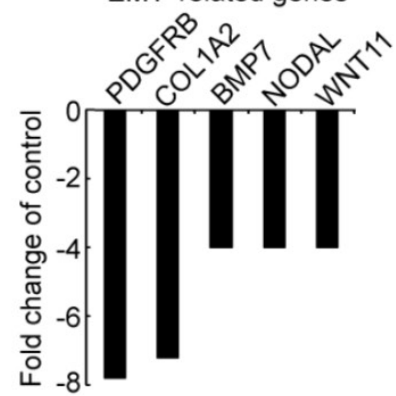

C

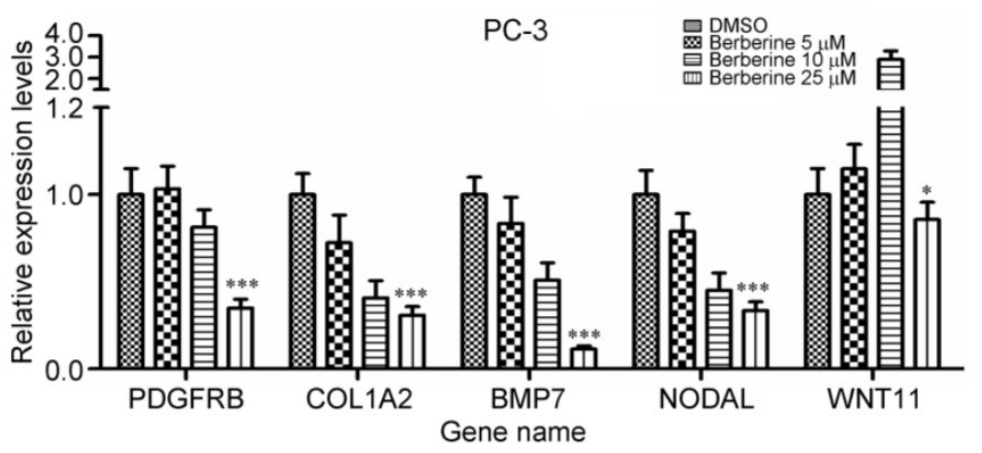

D

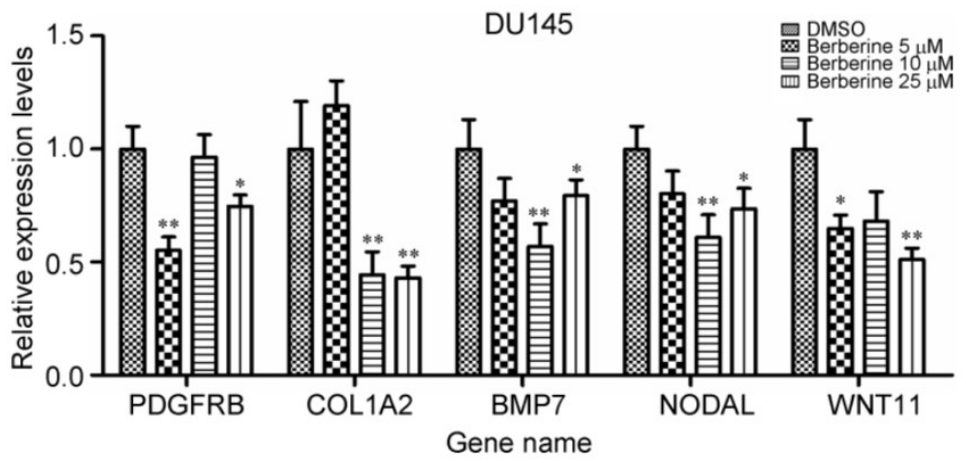

Figure 3. Berberine inhibits expressions of multiple genes associated with EMT activation. (A) Total RNA from untreated and berberine-treated ( $25 \mu M$ for $24 \mathrm{~h}$ ) PC- 3 cells was characterized using the Human EMT PCR Array. Figure shows scatter plot of the difference in relative transcript abundance of 84 key genes that either change their expression during the EMT process or regulate those EMT-related gene expression changes. (B) The top five transcripts exhibiting $>4$-fold downregulation are depicted as a proportion of all examined transcripts $24 \mathrm{~h}$ after berberine treatment compared to the control. Downregulated genes identified by EMT PCR Array experiments and verified by an independent quantitative RT-PCR of PC-3 (C) and DU145 (D) cells, respectively. A quantitative RT-PCR was done using independent primers to those used in the array experiments. Relative mRNA abundances were calculated using GAPDH as an endogenous control. Quantitative RT-PCR values are the mean \pm SEM of $n=3$ and normalized to the mean of untreated cells. ${ }^{*} p<0.05,{ }^{* *} p<0.01,{ }^{* * * *} p<0.001$.

\section{Ectopic activation of berberine-mediated suppression of two EMT-related genes in metastatic prostate tumors is a strong predictor of a poor prognosis}

To understand whether berberine-mediated suppression of EMT-related genes was upregulated in metastatic prostate cancer, we next investigated expressions of these genes in prostate tissues. We recruited a cohort of 140 prostate cancer patients from a publicly available dataset, deposited in the NCBI Gene Expression Omnibus (GEO) under accession no. GSE21032 (22). The samples included normal prostate tissues, primary prostate cancer tissues, and metastatic tissues. As shown in Figure 4, we observed that BMP7 (Figure 4A), NODAL (Figure 4B), and WNT11 (Figure 4C) expressions were significantly upregulated in metastatic prostate cancer tissues compared to the primary tumor group, whereas expressions of the PDGFRB and COL1A2 genes showed no statistically 
significant (NS) difference between the metastatic group and primary tumor group (Additional file 1: supplementary Figure 2). As noted above, the three upregulated genes of metastatic prostate cancer tissues were previously reported to be associated with the aggressive progression of prostate cancer $(21,23)$; however, the clinical relevance of these genes in prostate cancer is still unclear. This led us to explore the prognostic relevance of these EMT-related genes in prostate cancer using Kaplan-Meier survival analysis (24). A cohort of a transcriptome dataset of prostate cancer with outcomes was available from the Taylor database (22). Specifically, as shown in Figure $4 \mathrm{D}$, only the expression levels of the two EMT-related genes, BMP7 and NODAL, were able to stratify pa-
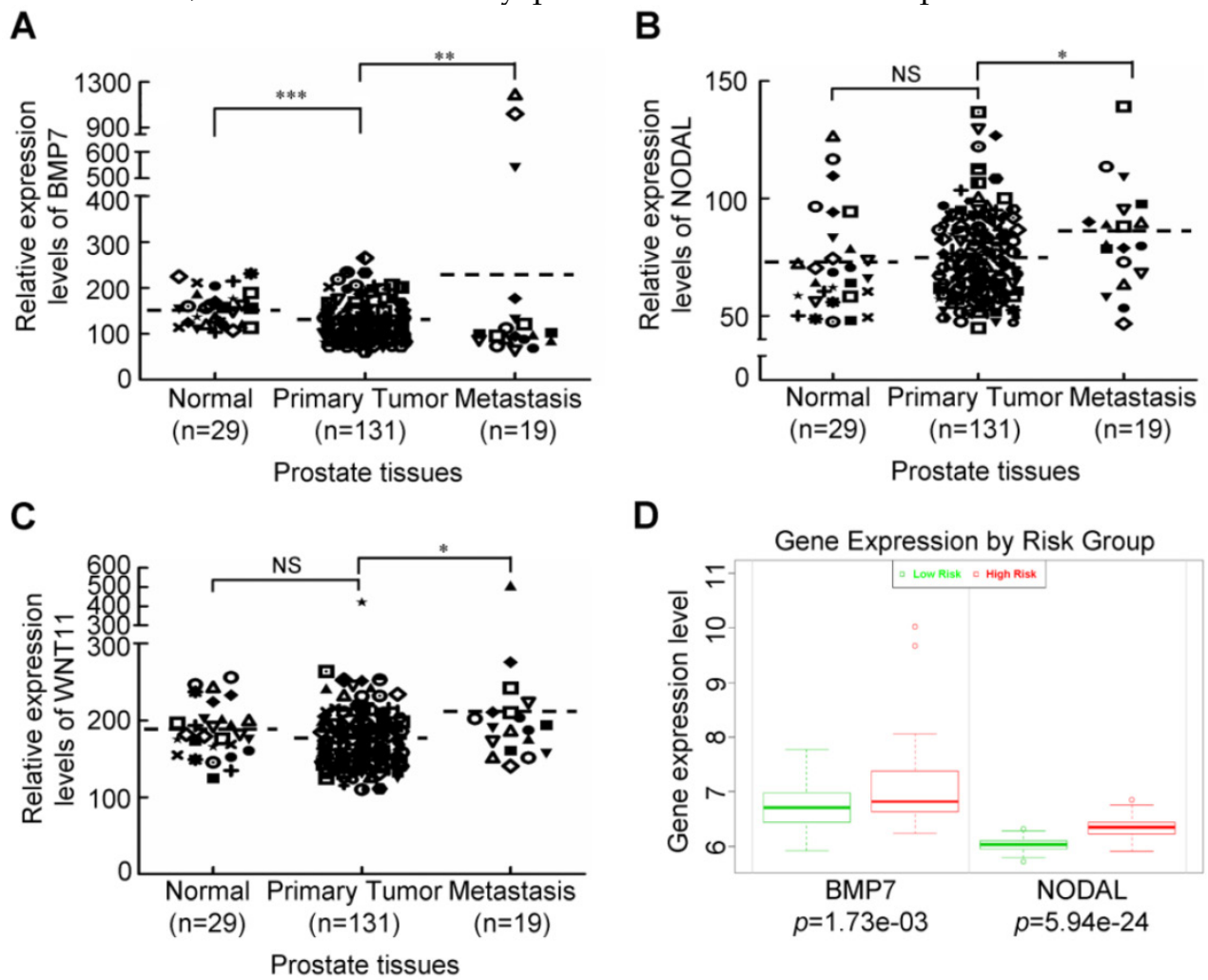

tients by K-means clustering into two subgroups (low- and high-risk groups) which exhibited significant differences $(p=0.0440)$ in overall survival according to the Kaplan-Meier survival analysis (Figure $4 \mathrm{E})$. However, there was no significant difference in genes selected by a single-gene or any combination of two- or three-gene models according to the Kaplan-Meier survival analysis (Additional file 1: supplementary Figure 3). Taken together, these data are remarkable in light of the high BMP7 and NODAL expressions of metastatic prostate cancer tissues with shorter survival times of prostate cancer patients and provide therapeutic interventions when the expressions of these genes rise following definitive treatment for advanced prostate cancer.

\section{B}

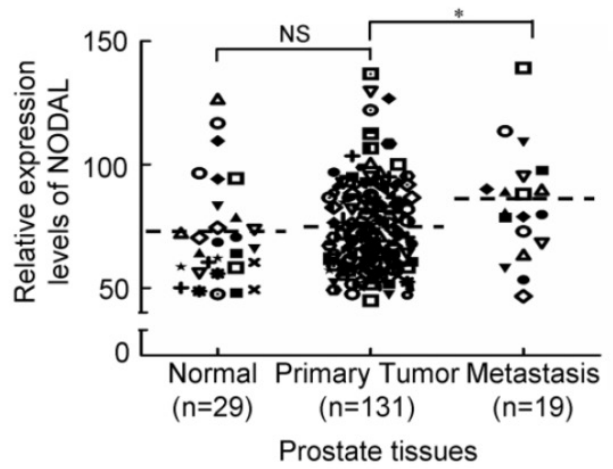

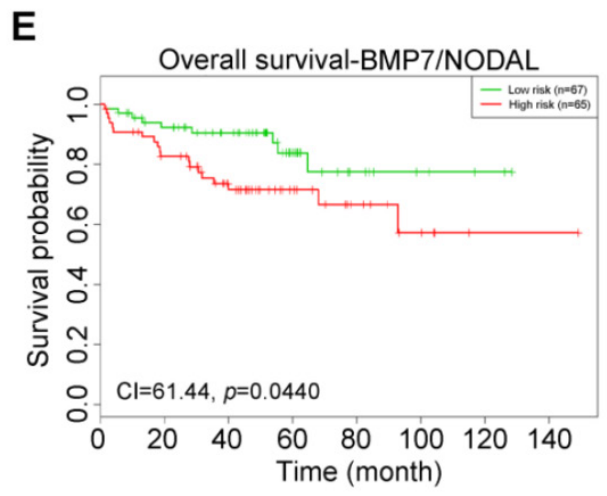

Figure 4. Aberrant expressions of berberine-modulates EMT-related genes associated with shorter survival of prostate cancer patients. Relative expression levels of BMP7 (A), NODAL (B), and WNT11 (C) in different clinical stages of prostate cancer tissues analyzed using the public GEO database. (D) Box plots comparing differences in BMP7 and NODAL gene expressions between risk groups using a $t$-test. (E) Two-gene (BMP7 and NODAL) combinations to accurately predict patient outcomes using Kaplan-Meier analyses of patients with high expressions of the two-gene set (high risk) ( $n=65)$ from Taylor et al.'s dataset showing stratification of BMP7 and NODAL for shorter-surviving versus longer-surviving patients. ${ }^{*} p<0.05,{ }^{* *} p<0.01,{ }^{* * *} p<0.001$ 


\section{Discussion}

Cancer cell metastasis is a complex and multistep process that includes cell migration, invasion, adhesion, and vessel formation (25). It has been demonstrated that about $90 \%$ of all cancer deaths can be linked to tumor metastasis (26). Therefore, it is important to identify an agent that could disrupt one or more steps of the metastatic process would be decrease the mortality of cancer patients. A number of natural products have been investigated with antimetastatic effect for prostate cancer cells (27-30). The present study established for the first time the important role played by the natural alkaloid, berberine, in repressing the metastatic process and the invasive ability of prostate cancer cells. There are several lines of evidence that support this conclusion which are listed below. First, our in vitro data clearly demonstrated that berberine had significant suppressive effects on the migration and invasion of highly metastatic prostate cancer cells. Second, berberine markedly decreased five EMT-related genes in a dose-dependent manner of PC-3 cells, and these genes are relevant to cancer metastasis. Third, berberine-mediated suppression of two high-expression EMT-related genes of metastatic prostate tumors was associated with a shorter survival of prostate cancer patients. Collectively, this study is the first to report that expressions of the BMP7 and NODAL genes are inhibited by berberine, and their aberrant expression together accurately predicted prostate cancer outcomes.

Several studies have shown that Snail is a prime promoter of metastasis in a variety of cancer types (31, 32). Recently, Whiteland et al. (33) has demonstrated Snail expression was significantly increased in prostate cancer tissues and was strongly associated with increasing clinical stage but did not demonstrate a significant association with patient survival. In the present study, we demonstrated that Snail expressions was significantly upregulated in metastatic prostate cancer tissues compared to the primary tumor group (Additional file 1: supplementary Figure 4A), and high expression of Snail in prostate cancer tissues was also not significantly associated with patient survival (Additional file 1: supplementary Figure 4B). However, there was more significant in genes combination of BMP7/NODAL/SNAI1 three-gene models than BMP7/NODAL two-gene models according to the Kaplan-Meier survival analysis (Additional file 1: supplementary Figure 4C compared to Figure 4E). Taken together, this results provide evidence of a three-gene prognostic model that can accurately define prostate cancer clinical risk groups and could thus assist therapeutic decisions in prostate cancer patients.
The EMT is characterized by the loss of cell-cell adhesion and an increase in cell motility, and it is a key process in cancer progression and metastasis, making EMT inhibition an attractive therapeutic strategy (34). It was demonstrated that berberine decreased A549 lung cancer cell migration and invasion in a dose-dependent manner through TGF- $\beta$-regulated EMT signaling (14). In this study, we also found that the migratory and invasive abilities of prostate cancer cells were inhibited by berberine through inhibition of TGF- $\beta$-related signaling molecules, such as BMP7, NODAL, and WNT11 (the expression of TGF- $\beta$ was downregulated by about 2 -fold after berberine treatment; data not shown). Effects of TGF- $\beta$ can locally result in greater tumor invasiveness through stimulation of angiogenesis or induction of the EMT (35). These results imply that berberine inhibits prostate cancer cell migration and invasion by suppressing the TGF- $\beta$-induced EMT.

Plastic phenotypes associated with aggressive tumors present a significant challenge in targeting cancer cells with EMT properties, which seems to be responsible for the morphologic and functional heterogeneity seen in cancers (36). Advances in the field of cancer research of the EMT have enabled characterization of the reemergence of specific embryonic signaling pathways, such as NODAL (37), thus contributing to our understanding of the molecular mechanisms that regulate cancer cell plasticity and aggressiveness. By understanding embryonic signaling pathways reactivated in many cancers and their contributions to tumor cell plasticity, new strategies will emerge about the suppression of this elusive phenotype (38). In our study, of the three validated metastatic oncogenes by EMT PCR Array and quantitative RT-PCR analysis, all of them may be a regulator of tumor cell plasticity and tumorigenecity. Because none of these three genes is highly expressed by primary prostate tissues but all are overexpressed by aggressive metastatic prostate tumor tissues (Figure 4A-C), they may represent valuable new therapeutic targets. Thus, the diagnostic potential of the combination of expression levels of these genes in human prostate carcinoma seems promising.

In summary, results from this study identified for the first time that berberine inhibits the migration and invasion of prostate cancer cells through inhibiting EMT-related genes. Measurement of EMT-related gene expression patterns may guide treatment choices for determining which patients are likely to benefit from berberine therapy. Among the EMT-related genes in the EMT PCR Array panel, BMP7, NODAL, and Snail can be incorporated into prognostic assays to monitor patients on active surveillance to facilitate appropriate courses of treatment. In addition, more 
mechanism-based studies are therefore needed to develop berberine as a pharmacologically safe agent alone or in combination with other antimetastatic drugs, for treating metastatic prostate cancer patients.

\section{Supplementary Material}

Additional File 1:

Supplementary Table and Supplementary Figures.

http://www.medsci.org/v12p0063s1.pdf

\section{Acknowledgements}

This project was funded by the National Science Council of Taiwan (NSC102-2320-B-038-007 to KHL), Taipei Medical University (TMU101-AE1-B19 to KHL) and Taipei Medical University-Shuang Ho Hospital (103TMU-SHH-05 to KHL). We thank Prof. YW Cheng, Prof. H Lee and Dr. YN Liu at the Graduate Institute of Cancer Biology and Drug Discovery, College of Medical Science and Technology, Taipei Medical University, Taipei, Taiwan, for their skillful technical assistance. We also acknowledge the technical support provided by the Instrument Center of the National Defense Medical Center, Taipei, Taiwan.

\section{Competing Interests}

The authors have declared that no competing interest exists.

\section{References}

1. Freedland SJ. Screening, risk assessment, and the approach to therapy in patients with prostate cancer. Cancer 2011; 117:1123-35.

2. Ye XC, Choueiri M, Tu SM, Lin SH. Biology and clinical management of prostate cancer bone metastasis. Frontiers in bioscience: a journal and virtual library 2007; 12:3273-86.

3. Thiery JP. Epithelial-mesenchymal transitions in tumour progression. Nature reviews Cancer 2002; 2:442-54.

4. Josson S, Nomura T, Lin JT, Huang WC, Wu D, Zhau HE, et al. beta2-microglobulin induces epithelial to mesenchymal transition and confers cancer lethality and bone metastasis in human cancer cells. Cancer research 2011; 71:2600-10.

5. Tillhon M, Guaman Ortiz LM, Lombardi P, Scovassi AI. Berberine: new perspectives for old remedies. Biochem Pharmacol 2012; 84: 1260-7.

6. Kuo HP, Chuang TC, Yeh MH, Hsu SC, Way TD, Chen PY, et al. Growth suppression of HER2-overexpressing breast cancer cells by berberine via modulation of the HER2/PI3K/Akt signaling pathway. Journal of agricultural and food chemistry 2011; 59:8216-24.

7. Lin CC, Yang JS, Chen JT, Fan S, Yu FS, Yang JL, et al. Berberine induces apoptosis in human HSC-3 oral cancer cells via simultaneous activation of the death receptor-mediated and mitochondrial pathway. Anticancer Res 2007; 27:3371-8.

8. Mantena SK, Sharma SD, Katiyar SK. Berberine inhibits growth, induces G1 arrest and apoptosis in human epidermoid carcinoma A431 cells by regulating Cdki-Cdk-cyclin cascade, disruption of mitochondrial membrane potential and cleavage of caspase 3 and PARP. Carcinogenesis 2006; 27:2018-27.

9. Lin JP, Yang JS, Wu CC, Lin SS, Hsieh WT, Lin ML, et al. Berberine induced down-regulation of matrix metalloproteinase-1, -2 and -9 in human gastric cancer cells (SNU-5) in vitro. In vivo 2008; 22:223-30.

10. Li H, Guo L, Jie S, Liu W, Zhu J, Du W, et al. Berberine inhibits SDF-1-induced AML cells and leukemic stem cells migration via regulation of SDF-1 level in bone marrow stromal cells. Biomedicine \& pharmacotherapy Biomedecine \& pharmacotherapie 2008; 62:573-8.

11. Ho YT, Yang JS, Li TC, Lin JJ, Lin JG, Lai KC, et al. Berberine suppresses in vitro migration and invasion of human SCC-4 tongue squamous cancer cells through the inhibitions of FAK, IKK, NF-kappaB, u-PA and MMP-2 and -9. Cancer letters 2009; 279:155-62.

12. Yan L, Yan K, Kun W, Xu L, Ma Q, Tang Y, et al. Berberine inhibits the migration and invasion of T24 bladder cancer cells via reducing the expression of heparanase. Tumour biology : the journal of the International Society for Oncodevelopmental Biology and Medicine 2013; 34:215-21.
13. Zhang LJ, Zhang LJ, Quan W, Wang BB, Shen BL, Zhang TT, et al. Berberine inhibits HEp-2 cell invasion induced by Chlamydophila pneumoniae infection. Journal of microbiology 2011; 49:834-40.

14. Qi HW, Xin LY, Xu X, Ji XX, Fan LH. Epithelial-to-mesenchymal transition markers to predict response of Berberine in suppressing lung cancer invasion and metastasis. Journal of translational medicine 2014;12:22.

15. Lee KH, Hsu EC, Guh JH, Yang HC, Wang D, Kulp SK, et al. Targeting energy metabolic and oncogenic signaling pathways in triple-negative breast cancer by a novel adenosine monophosphate-activated protein kinase (AMPK) activator. The Journal of biological chemistry 2011; 286:39247-58.

16. Guh JH, Chang WL, Yang J, Lee SL, Wei S, Wang D, et al. Development of novel adenosine monophosphate-activated protein kinase activators. Journal of medicinal chemistry 2010; 53:2552-61.

17. Hsieh IS, Chang KC, Tsai YT, Ke JY, Lu PJ, Lee KH, et al. MicroRNA-320 suppresses the stem cell-like characteristics of prostate cancer cells by downregulating the $\mathrm{Wnt} /$ beta-catenin signaling pathway. Carcinogenesis 2013; 34:530-8.

18. Harma V, Virtanen J, Makela R, Happonen A, Mpindi JP, Knuuttila M, et al. A comprehensive panel of three-dimensional models for studies of prostate cancer growth, invasion and drug responses. PloS one 2010;5:e10431.

19. Webb BA, Chimenti M, Jacobson MP, Barber DL. Dysregulated pH: a perfect storm for cancer progression. Nature reviews Cancer 2011; 11:671-7.

20. Uysal-Onganer P, Kawano Y, Caro M, Walker MM, Diez S, Darrington RS, et al. Wnt-11 promotes neuroendocrine-like differentiation, survival and migration of prostate cancer cells. Molecular cancer 2010; 9:55.

21. Lawrence MG, Margaryan NV, Loessner D, Collins A, Kerr KM, Turner M, et al. Reactivation of embryonic nodal signaling is associated with tumor progression and promotes the growth of prostate cancer cells. The Prostate 2011; 71:1198-209.

22. Taylor BS, Schultz N, Hieronymus H, Gopalan A, Xiao Y, Carver BS, et al. Integrative genomic profiling of human prostate cancer. Cancer cell 2010; 18:11-22.

23. Morrissey C, Brown LG, Pitts TE, Vessella RL, Corey E. Bone morphogenetic protein 7 is expressed in prostate cancer metastases and its effects on prostate tumor cells depend on cell phenotype and the tumor microenvironment. Neoplasia 2010; 12:192-205.

24. Aguirre-Gamboa R, Gomez-Rueda H, Martinez-Ledesma E, Martinez-Torteya A, Chacolla-Huaringa R, Rodriguez-Barrientos A, et al. SurvExpress: an online biomarker validation tool and database for cancer gene expression data using survival analysis. PloS one 2013; 8:e74250.

25. Bellahcene A, Castronovo V, Ogbureke KU, Fisher LW, Fedarko NS. Small integrin-binding ligand N-linked glycoproteins (SIBLINGs): multifunctional proteins in cancer. Nature reviews Cancer 2008; 8:212-26.

26. Mehlen P, Puisieux A. Metastasis: a question of life or death. Nature reviews Cancer 2006; 6:449-58.

27. Shin Y, Kim GD, Jeon JE, Shin J, Lee SK. Antimetastatic effect of halichondramide, a trisoxazole macrolide from the marine sponge Chondrosia corticata, on human prostate cancer cells via modulation of epithelial-to-mesenchymal transition. Marine drugs 2013; 11:2472-85.

28. Lieberman R. Chemoprevention of prostate cancer: current status and future directions. Cancer metastasis reviews 2002; 21:297-309.

29. Zhang X, Haney KM, Richardson AC, Wilson E, Gewirtz DA, Ware JL, et al. Anibamine, a natural product CCR5 antagonist, as a novel lead for the development of anti-prostate cancer agents. Bioorganic \& medicinal chemistry letters 2010; 20:4627-30.

30. Yao H, Wang H, Zhang Z, Jiang BH, Luo J, Shi X. Sulforaphane inhibited expression of hypoxia-inducible factor-1alpha in human tongue squamous cancer cells and prostate cancer cells. International journal of cancer Journal international du cancer 2008; 123:1255-61.

31. Osafune K, Takasato M, Kispert A, Asashima M, Nishinakamura R. Identification of multipotent progenitors in the embryonic mouse kidney by a novel colony-forming assay. Development 2006; 133:151-61.

32. Becker KF, Rosivatz E, Blechschmidt K, Kremmer E, Sarbia M, Hofler H. Analysis of the E-cadherin repressor Snail in primary human cancers. Cells, tissues, organs 2007; 185:204-12.

33. Whiteland H, Spencer-Harty S, Thomas DH, Davies C, Morgan C, Kynaston $\mathrm{H}$, et al. Putative prognostic epithelial-to-mesenchymal transition biomarkers for aggressive prostate cancer. Experimental and molecular pathology 2013; 95:220-6.

34. Lim J, Thiery JP. Epithelial-mesenchymal transitions: insights from development. Development 2012; 139:3471-86.

35. Miyazono K. Transforming growth factor-beta signaling in epithelial-mesenchymal transition and progression of cancer. Proceedings of the Japan Academy Series B, Physical and biological sciences 2009; 85:314-23.

36. Bao B, Ahmad A, Azmi AS, Ali S, Sarkar FH. Overview of cancer stem cells (CSCs) and mechanisms of their regulation: implications for cancer therapy. Current protocols in pharmacology 2013; 61:1-14.

37. Strizzi L, Hardy KM, Seftor EA, Costa FF, Kirschmann DA, Seftor RE, et al. Development and cancer: at the crossroads of Nodal and Notch signaling. Cancer research 2009; 69:7131-4.

38. Strizzi L, Hardy KM, Kirschmann DA, Ahrlund-Richter L, Hendrix MJ. Nodal expression and detection in cancer: experience and challenges. Cancer research 2012; 72:1915-20. 